\title{
Identification and Role of Regulatory Non-Coding RNAs in Listeria monocytogenes
}

\author{
Benjamin Izar ${ }^{1,2}$, Mobarak Abu Mraheil ${ }^{1}$ and Torsten Hain ${ }^{1, *}$
}

1 Institute of Medical Microbiology, Justus-Liebig-University, Frankfurter Strasse 107, Giessen 35392, Germany; E-Mails: Benjamin.Izar@med.uni-giessen.de (B.I.);

Mobarak.Mraheil@mikrobio.med.uni-giessen.de (M.A.M.)

2 Department of Internal Medicine, Massachusetts General Hospital, 55 Fruit Street, Boston, MA 02118, USA

* Author to whom correspondence should be addressed;

E-Mail: Torsten.Hain@mikrobio.med.uni-giessen.de; Tel.: +49-641-985-46400; Fax: +49-641-985-46409.

Received: 28 June 2011; in revised form: 15 July 2011 / Accepted: 8 August 2011 / Published: 10 August 2011

\begin{abstract}
Bacterial regulatory non-coding RNAs control numerous mRNA targets that direct a plethora of biological processes, such as the adaption to environmental changes, growth and virulence. Recently developed high-throughput techniques, such as genomic tiling arrays and RNA-Seq have allowed investigating prokaryotic cis-and trans-acting regulatory RNAs, including sRNAs, asRNAs, untranslated regions (UTR) and riboswitches. As a result, we obtained a more comprehensive view on the complexity and plasticity of the prokaryotic genome biology. Listeria monocytogenes was utilized as a model system for intracellular pathogenic bacteria in several studies, which revealed the presence of about 180 regulatory RNAs in the listerial genome. A regulatory role of non-coding RNAs in survival, virulence and adaptation mechanisms of L. monocytogenes was confirmed in subsequent experiments, thus, providing insight into a multifaceted modulatory function of $\mathrm{RNA} / \mathrm{mRNA}$ interference. In this review, we discuss the identification of regulatory RNAs by high-throughput techniques and in their functional role in L. monocytogenes.
\end{abstract}


Keywords: Listeria monocytogenes; sRNA; asRNA; riboswitch; non-coding RNA; regulatory RNA; RNA-Seq; whole genome tiling arrays; infection; UTR

\section{Introduction}

Listeria monocytogenes is a Gram-positive facultative intracellular bacterium that occurs ubiquitously in nature. Its genome bears a low G + C DNA content and is closely related to the genus of Streptococcus, Staphylococcus, Enterococcus and Clostridium. Eight different species have been described for the Listeria genus, including Listeria monocytogenes, Listeria ivanovii, Listeria innocua, Listeria welshimeri, Listeria seeligeri, Listeria grayi, Listeria marthii and the recently isolated Listeria rocourtiae [1-4].

L. monocytogenes cause infections in humans and animals, such as meningitis and septicaemia that are associated with a high mortality rate up to $30 \%$, despite antibiotic therapy [5]. A characteristic feature in the pathogenesis of L. monocytogenes is the ability to actively induce its own phagocytosis by host cells mediated by internalins (inl) and escape from the phagosom/autophagosom using the virulence factors listerolysin (LLO) or ActA for actin polymerization. Once located in the host cytosol, it replicates and induces actin-based motion allowing the pathogen to move in infected cells and spread to adjacent cells [3]. With exception of $i n l A-C$ and $h p t$ [6], the major virulence genes, responsible for the intracellular life cycle of $L$. monocytogenes are clustered in a $\sim 9 \mathrm{~kb}$ chromosomal region that is controlled by the principal virulence regulator PrfA [2,3,7].

L. monocytogenes has served as a model pathogen for the investigation of host-pathogen interactions and the immune response following infection intracellular bacteria and is now one of the best characterized microorganisms worldwide. Using recently developed high-throughput techniques, such as tiling arrays and next-generation DNA sequencing, which allows high-throughput RNA analysis through cDNA sequencing (RNA-Seq), several studies uncovered numerous non-coding RNAs in the listerial genome that were expressed under different conditions and introduce a new chapter of L. monocytogenes as an indispensable model organism for the investigation of prokaryotic transcriptomics [8-10].

Three major studies detected 180 regulatory RNAs that are almost exclusively non-coding [8-10]. Discovery of sRNAs in several other bacteria, including Escherichia coli [11,12], Salmonella typhimurium [13,14], Pseudomonas aeruginosa [15], Streptococcus pyogenes [16-18], Streptococcus pneumoniae [19] and Staphylococcus aureus [20] extended our view to an unexpected complexity of prokaryotic genomes and transcriptomic regulation. These studies also emphasized that the portion of non-coding RNAs was much higher than previously expected [21].

Non-coding RNAs control the fate of mRNA through different mechanisms and thereby influence several biological processes and virulence in L. monocytogenes. In general, interference of regulatory RNA with mRNA leads to destabilization and degradation of the target mRNA [22]. Interaction generally occurs through (i) trans-acting sRNAs encoded in intergenic regions (IGRs) at a chromosomal locus distal to the target transcript, (ii) anti-sense RNA (asRNA) encoded on the opposite strand of the open reading frame (ORF) or overlapping 5' or 3' untranslated region (UTR), and (iii) cis-regulatory RNAs, including riboswitches and 5'UTRs located upstream of a target gene 
with possible overlap with the ORF [23]. However, bifunctional regulatory RNAs, such as the SAM riboswitch that also acts as trans-acting sRNA (discussed below), have recently been described and highlight an unexpected level of complexity of prokaryotic transcriptional regulation [24].

In this paper, we give an overview on non-coding RNAs in L. monocytogenes, their role in virulence and adaptation to environmental changes predominantly identified by genomewide approaches.

\section{Identification of Regulatory RNAs in L. monocytogenes Using High-Throughput Techniques}

Gene expression analyses using microarrays provided us with comprehensive information on how L. monocytogenes adjusts to physiological and hostile environmental changes. For instance, previous studies investigated the intracellular expression signature and found that Listeria induces several metabolic and virulence genes that in concert allow survival and proliferation within host cells $[25,26]$. Further studies using mutant bacteria lacking transcription factor SigmaB $\left(\sigma^{\mathrm{B}}\right)$ characterized several stress response genes that are important for intracellular survival of the pathogen [27,28]. However, due to the microarray chip design and technology, only intensities of transcripts from protein-coding regions are assessed and leave non-coding regions unexamined.

Two innovative techniques, genomic tiling arrays and RNA-Seq have revolutionized the field of RNA research, because they provide an uninterrupted picture of the whole transcriptome, including non-coding RNAs $[9,10,13,29,30]$. In both cases, isolated RNA is reverse transcribed to cDNA. Prior to reverse transcription in RNA-Seq, mRNA enrichment may be performed by reducing rRNAs and tRNAs levels from the total RNA sample [21]. In RNA-Seq, cDNA is then subjected to high-throughput sequencing. Resulting sequences are mapped to the genome and deliver a detailed snapshot of the current transcriptome. Whole genomic tiling array are microarray that cover the entire genome, regardless of the position of annotated ORFs, by overlapping oligonucleotide probes and permits the identification of non-coding regions. cDNA is hybridized to the array to measure intensity of each transcript which then allows to analyze expression profiles.

The first sRNA in L. monocytogenes has been identified by Barry et al. in 1999 [31]. Subsequently, additional 20 sRNAs were identified using northern blots and co-immunoprecipitation using Hfq, a protein that binds to sRNAs and stabilizes the hybridization to target mRNAs [32-34].

Using whole genome tiling arrays, Toledo-Arana et al. provided the first genome-wide study on the identification of regulatory RNA candidates expressed in L. monocytogenes and its deletion mutants under various physiological growth conditions in human blood, the intestinal lumen and other organs in mice [9]. They identified 103 putative regulatory RNA elements, several of which are absent in the non-pathogenic L. innocua. These data was largely confirmed by a following study using RNA-Seq by Oliver et al. who found 67 non-coding RNAs, of which 7 have not been described previously [8]. One trans-acting sRNA, $s b r E$ was found to be directly regulated by $\sigma^{\mathrm{B}}$. However, due to the RNA-Seq protocol, regulatory RNAs previously described to be coded anti-sense were not covered. In a more recent study, however, Hain and colleges provided the so far most detailed picture on sRNAs in L. monocytogenes using RNA-Seq that also allowed for directional reads [10]. 150 small non-coding RNA elements were found, which enclosed 75\% [9] and 69\% [8], respectively, of previously reported regulatory RNAs. Differences in the number of regulatory RNAs was mainly due to 25 newly discovered asRNAs by Mraheil et al. [10]. Furthermore, comparison with a computational analysis for 
the prediction of regulatory RNAs in L. monocytogenes revealed that only $33 \%$ of predicted sRNAs overlapped with the RNA-Seq data [10,35].

\section{How Non-Coding Regulatory RNAs Control the Fate of mRNA in L. monocytogenes}

L. monocytogenes survives at a broad range of temperatures $\left(0-45^{\circ} \mathrm{C}\right)$ and $\mathrm{pH}(4.5-9)$, at high salt concentrations $(10 \% \mathrm{NaCl})$ and also in most hostile environment, such as the phagocytic vacuole and within infected macrophages $[2,3,10]$. For this reason, sensing the surrounding and rapid adaptation to environmental changes is essential for the bacterium in order to survive in its ecological niches. Both tasks may be accomplished by non-coding regulatory RNAs by interfering with different structures of the target transcript or due to regulation at the transcriptional level. Compared to regulatory proteins, RNAs offer some advantages, because they present a rapid connection between quorum sensing and direct destabilization of target mRNA, and allow a fine tuning of the response at a post-transcriptional level. In addition, unnecessary regulatory RNAs may be cleared quickly using less energy. Therefore, it is not surprising that several bacteria maintain a considerable set of regulatory RNAs that account for at least $3-13 \%$ of the whole genome [21].

\section{5'UTRs and Multi-Functional Regulatory RNAs}

5'UTRs are defined as the mRNA region starting at the promoter and ends with the start codon of the ORF. Since the transcription of an ORF may begin at different promoters, the length of the 5'UTR of a protein coding transcript may vary in length. 5'UTRs act as sensors for temperature and metabolites in L. monocytogenes and affect virulence and growth at the same time.

PrfA, the master virulence regulator in L. monocytogenes, is controlled by a thermosensor that is located 116 nucleotides 5'UTR upstream of the coding mRNA sequence [36]. At low temperatures (30 ${ }^{\circ} \mathrm{C}$ ), the $5^{\prime}$ UTR forms a complex secondary structure that hides the Shine-Dalgarno (SD) region and thus prevents the translation of $\operatorname{prfA}$. An increase in temperature to $37{ }^{\circ} \mathrm{C}$ leads to formation of an alternative secondary structure that uncovers the SD site and allows for translation of the prfA transcript. Subsequently, PrfA induces expression of e.g. the major virulence factors LLO and ActA.

Metabolite-binding 5'UTRs that act as sensors for a substrate are also known as riboswitches. These RNA control elements possess an aptamer, an RNA region that is specific to a metabolite (e.g., lysine). Binding of the metabolite leads to an alteration of the riboswitch structure and results in transcription termination of the downstream gene. Several putative riboswitches have been identified for $L$. monocytogenes [9,10,24,37], of which the lysine riboswitch (LysRS) is one of the best characterized. The LysRS is located 280 nucleotides 5'UTR upstream of a lysine transporter gene (lmo0798). When present in abundance, lysine binds to the LysRS and stabilizes its tertiary structure leading to the formation of a Rho-independent transcriptional terminator (RITT) within the LysRS that prevents the expression of the downstream gene. RITT are strong secondary RNA structures that destabilize the binding of the RNA-polymerase to target DNA and thus stop transcription of downstream genes. In the absence of lysine, an anti-terminator structure is formed within the LysRS, which then allows for transcription of the lysine transporter gene. Interestingly, Toledo-Arana et al. discovered that LysRS regulates expression of the upstream protein coding gene lmo0799, thus, functions as a 3'UTR regulator as well [8]. The transcript of lmo0799 lacks an intrinsic 3'end terminator structure other than 
the LysRS that forms the RITT in lysine rich conditions. In contrast, lysine depletion leads to generation of a long transcript consisting of lmo0799-LysRS-lmo0798 [9]. Additional regulator elements with a dual 5' and 3'UTR function have been identified in L. monocytogenes, including the cobalamin, T-box, M-box and S-adenosylmethionine binding (SAM) riboswitches [9]. Furthermore, a recent study discovered that two SAM riboswitches can also function as trans-acting sRNA [24]. SAM-binding leads to formation of a terminator structure, also known as SAM riboswitch element A (SreA), which blocks expression of downstream genes lmo2419, lmo2418 and lmo2417 that are involved in the cysteine or methionine metabolism. Strikingly, SreA also diffuses and binds to the 5'UTR of the prfA mRNA preventing its translation. Because the 5'UTR thermosensor of prfA exhibits a closed stem-loop structure at low temperatures, thus hiding the SD site, binding of SreA to $\operatorname{prf} A$ predominantly occurred at $37{ }^{\circ} \mathrm{C}$, when the $\operatorname{prf} A$ thermosensor exhibits its open structure and allows SreA to target the SD region. These findings outline the complexity of the prokaryotic regulation by non-coding RNAs that are rather multifunctional and emphasize the potential flexibility of how bacteria control the expression of essential virulence genes.

Furthermore, a regulatory role in the connection between nutrient availability and growth was recently shown for the flavin mononucleotide (FMN) riboswitch [37]. Addition of roseoflavin, a riboflavin analog, which is the natural ligand of the FMN riboswitch, inhibited the expression of the downstream riboflavin transporter gene, but also drastically limited growth of Listeria. In contrast, mutation of the FMN riboswitch allowed the pathogen to proliferate in the presence of roseoflavin. In conclusion, this study provides evidence that the FMN riboswitch directly controls bacterial growth.

\section{Trans-Acting sRNAs}

Trans-acting small non-coding RNAs (sRNAs) are encoded in intergenic regions (IGR) distal to the target gene. To date, 101 sRNAs (including SreA) have been identified in L. monocyotenes, predominantly by using whole genome tiling-arrays and RNA-Seq [8-10]. However, this number may be underestimated since it was discovered that riboswitches can also act as trans-acting sRNAs [24]. The entire set of 21 sRNAs previously discovered by other methods was confirmed by whole genome tiling arrays [9]. With a few exceptions, sRNAs usually measure less than 500 nucleotides in length, while most are ranging between 50-150 nucleotides.

Although functional data on sRNAs is limited, recently some sRNAs, including rliB, rli31, rli33-1, rli38 and rli50 were shown to have a role in virulence and growth of L. monocytogenes $[9,10]$. Except for rli31, these sRNAs are absent in L. innocua and other apathogenic strains of Listeria. A rli38 knockout strain displayed attenuated growth in several mouse organs [9]. $\Delta$ rli31, $\Delta$ rli33-1 and $\Delta r l i 50^{*}$ (due to an overlap between rli50 and rli112 in the intergenic region, the authors decided to delete rli50 and the distal part of rlil12 to create $\Delta r l i 50^{*}$ ) had decreased survival rates in infected murine macrophages, mouse organs and in an invertebrate infection model, when compared to the wild type strain, indicating an important role in virulence [10]. $\Delta r l i B$ displayed affected virulence, although it colonized the liver faster than the wild type strain [9].

29 sRNAs were shown to be exclusively transcribed by bacteria within infected host cells, indicating a role in response to intracellular stress [10]. Interestingly, in Listeriae grown in blood, five 
of these sRNAs (LhrC1-5) displayed expression patterns similar to that of the virulence gene locus and virulence genes vip (lmo0320), uhpT and inlC, suggesting a possible role in virulence regulation [9].

The complexity of regulatory networks by sRNAs is increased by potential interactions between sRNAs [9]. Considering, that translation of a certain mRNA may only occur when mRNA levels prevail over levels of its regulatory RNA, sRNA/sRNA interactions could partially neutralize destabilizing effects of regulatory RNAs and favour mRNA translation. Reliable target prediction of sRNAs and experimental confirmation is therefore essential and currently an intensively investigated field.

\section{Anti-Sense RNAs}

Anti-sense RNAs (asRNAs) are encoded on the complementary DNA strand to the target transcript. Among asRNAs, different types and mechanisms of action can be distinguished depending on the localization on the opposite strand: (i) asRNAs that cover one or more ORFs without overlap to the flanking regions and (ii) 5' or 3'UTRs of an mRNA overlapping with the mRNA encoded on the opposite strand.

In the first case, promoter as well as terminator of the asRNA is located in a region between the ORFs located at the opposite strand. The length of asRNA substantially varies and may range from a few bases to kilobases. Certain asRNAs may target only the SD region of a transcript while others cover several ORFs.

Overlapping 5'UTRs occur when the promoter of a certain gene is located within the targeted opposite divergent ORFs, but transcription of this gene ends upstream of the target ORF promoter. Overlapping 3'UTRs are produced when the promoter of the asRNA lies downstream of the target ORF terminator region and the transcription of the asRNA terminates within the ORF of the opposite strand.

More than 50 anti-sense regulatory RNAs and overlapping UTRs have been discovered in L. monocytogenes $[9,10]$. Although the majority of asRNAs identified so far are less than 150 nucleotides in size and represent bona fide asRNAs, several large UTRs have also been discovered. Functional and mechanistic data on the role of asRNAs is very limited. 3 asRNAs were confirmed by northern blots [9]. Interestingly, these asRNAs cover more than one ORF. For instance, the promoter of asRNA anti2095 lies on the opposite strand of lmo2095 and covers, both lmo2096 and lmo2097, which encode for hypothetical proteins that may be implicated in carbohydrate metabolism. A similar organization was observed for anti2325 and anti2394 [9].

Furthermore, the genome of L. monocytogenes harbours 13 overlapping 3'UTRs (anti0360, anti0945, anti0946, anti2260 [10] and anti0111, anti0734, anti1909, anti1910, anti1980, anti1981, anti2044, anti2045 and anti2259 [9]) as well as 7 overlapping 5'UTRs (anti0943, anti0977, anti2224 [10] and anti0306, anti0647, anti0648 and $\operatorname{mog} R$ [9]). So far, only $\operatorname{mog} R$ has been characterized in further detail. MogR is a transcriptional repressor of flagellum genes lmo0675, lmo0676 and lmo0677. $\operatorname{mog} R$ possesses two promoters, $\mathrm{P} 1$, which is localized 1697 nucleotides from the start codon and P2 at 45 nucleotides from the ATG. Transcription start at P1 generates a long overlapping 5'UTR that covers three genes required for flagellum synthesis in L. monocytogenes, thus decreasing pathogen motility. In line with this, overexpression of the long $m o g R$ transcript was shown 
to reduce the motility of $L$. monocytogenes. Interestingly, $\mathrm{P} 1$ also contains a $\sigma^{\mathrm{B}}$ box. A subsequent experiment with a $\sigma^{\mathrm{B}}$ isogenic deletion mutant showed that expression of the long anti-sense transcript starting at $\mathrm{P} 1$ was directly regulated by $\sigma^{\mathrm{B}}$. Consequently, $\sigma^{\mathrm{B}}$ dependent $m o g R$ induction at $\mathrm{P} 1$ resulted in the generation of a long $\operatorname{mog} R$ transcript resulting in decreased motility of L. monocytogenes [9]. This mechanism emphasizes a possible role of regulatory RNAs in the ability to colonize infected host organs.

\section{Role of Hfq and Transcriptional Control of Regulatory RNAs}

Interaction of trans-acting sRNAs with target mRNA often requires the RNA chaperone Hfq [23]. This protein increases the stability of sRNA/mRNA hybridizations by an unknown mechanism. In contrast to other Gram-positive bacteria Hfq has an important role in virulence of L. monocytogenes, whereas Hfq is commonly indispensable for virulence in Gram-negative pathogens. However, to date, homologues of Hfq have been found in only half of the sequenced bacteria. Although Hfq was shown to be required for target interaction of several sRNAs, such as LhrA-C, others interact with the target mRNA independently from Hfq [38]. Possibly, further RNA binding proteins are involved in binding stabilization of sRNAs as suggested for other intracellular bacteria [39].

Regulatory RNAs undergo transcriptional deregulation in a variety of conditions, most prominently in bacteria grown in blood and bacteria located in the intestinal lumen or within macrophages $[9,10]$. Transcriptional regulation of several sRNAs was shown to be mediated by $\sigma^{\mathrm{B}}$, while PrfA and Hfq had little or no effect on sRNA expression [8,9].

\section{Concluding Remarks and Future Outlook}

Identification of a number of non-coding regulatory RNAs in many prokaryotic genomes has widened our view on the complexity of transcription regulation in bacteria. Recent developments in high-throughput techniques have been instrumental in this process. Tiling arrays and RNA-Seq have not only led to the detection of the vast majority of today's known regulatory non-coding RNAs, but also channelled the discovery of new genes, the construction of the first operon map, the definition of untranslated regions and finally, the correction of previously annotated genes.

Although some insights have been obtained about how regulatory RNAs control mRNAs, precise functions and mechanisms remain largely unclear and need to be elucidated in further detail. Considering that only two years ago, the first sRNA has been described to have a role in virulence in Listeria, it becomes obvious that knowledge about the function of hundreds of regulatory RNAs and their target transcripts will have a remarkable impact on our understanding about several bacterial phenomena. For this process, L. monocytogenes serves as an unmatched model pathogen. Finally, regulatory RNAs may be attractive therapeutical targets to aim for with novel antibiotic treatments, such as PNAs $[9,40]$ or possibly serve as diagnostic parameters.

\section{Conflict of Interest}

The authors declare no conflict of interest. 


\section{Acknowledgments}

This work was supported by grants from the German Federal Ministry of Education and Research (BMBF ERA-NET Pathogenomics Network to the sncRNAomics project (62080061) to T.H.

\section{References}

1. Graves, L.M.; Helsel, L.O.; Steigerwalt, A.G.; Morey, R.E.; Daneshvar, M.I.; Roof, S.E.; Orsi, R.H.; Fortes, E.D.; Milillo, S.R.; den Bakker, H.C.; et al. Listeria marthii sp. nov., isolated from the natural environment, Finger Lakes National Forest. Int. J. Syst. Evol. Microbiol. 2010, 60, 1280-1288.

2. Hain, T.; Chatterjee, S.S.; Ghai, R.; Kuenne, C.T.; Billion, A.; Steinweg, C.; Domann, E.; Karst, U.; Jansch, L.; Wehland, J.; et al. Pathogenomics of Listeria spp. Int. J. Med. Microbiol. 2007, 297, 541-557.

3. Vazquez-Boland, J.A.; Kuhn, M.; Berche, P.; Chakraborty, T.; Dominguez-Bernal, G.; Goebel, W.; Gonzalez-Zorn, B.; Wehland, J.; Kreft J. Listeria pathogenesis and molecular virulence determinants. Clin. Microbiol. Rev. 2001, 14, 584-640.

4. Leclercq, A.; Clermont, D.; Bizet, C.; Grimont, P.A.; Le Fleche-Mateos, A.; Roche, S.M.; Buchrieser, C.; Cadet-Daniel, V.; Le, M.A.; Lecuit, M.; Allerberger, F. Listeria rocourtiae sp. nov. Int. J. Syst. Evol. Microbiol. 2010, 60, 2210-2214.

5. Hof, H.; Szabo, K.; Becker B. Epidemiology of listeriosis in Germany: a changing but ignored pattern. Dtsch. Med. Wochenschr. 2007, 132, 1343-1348.

6. Chico-Calero, I.; Suarez, M.; Gonzalez-Zorn, B.; Scortti, M.; Slaghuis, J.; Goebel, W.; Vazquez-Boland, J.A. Hpt, a bacterial homolog of the microsomal glucose- 6-phosphate translocase, mediates rapid intracellular proliferation in Listeria. Proc. Natl. Acad. Sci. USA 2002, 99, 431-436.

7. Cossart, P.; Toledo-Arana, A. Listeria monocytogenes, a unique model in infection biology: An overview. Microbes Infect. 2008, 10, 1041-1050.

8. Oliver, H.F.; Orsi, R.H.; Ponnala, L.; Keich, U.; Wang, W.; Sun, Q.; Cartinhour, S.W.; Filiatrault, M.J.; Wiedmann, M.; Boor, K.J. Deep RNA sequencing of L. monocytogenes reveals overlapping and extensive stationary phase and sigma B-dependent transcriptomes, including multiple highly transcribed noncoding RNAs. BMC Genomics 2009, 10, 641.

9. Toledo-Arana, A.; Dussurget, O.; Nikitas, G.; Sesto, N.; Guet-Revillet, H.; Balestrino, D.; Loh, E.; Gripenland, J.; Tiensuu, T.; Vaitkevicius, K.; et al. The Listeria transcriptional landscape from saprophytism to virulence. Nature 2009, 459, 950-956.

10. Mraheil, M.A.; Billion, A.; Mohamed, W.; Mukherjee, K.; Kuenne, C.; Pischimarov, J.; Krawitz, C.; Retey, J.; Hartsch, T.; Chakraborty, T.; et al. The intracellular sRNA transcriptome of Listeria monocytogenes during growth in macrophages. Nucleic Acids Res. 2011, 39, 4235-4248.

11. Zhang, A.; Wassarman, K.M.; Rosenow, C.; Tjaden, B.C.; Storz, G.; Gottesman, S. Global analysis of small RNA and mRNA targets of Hfq. Mol. Microbiol. 2003, 50, 1111-1124. 
12. Vogel, J.; Bartels, V.; Tang, T.H.; Churakov, G.; Slagter-Jager, J.G.; Huttenhofer, A.; Wagner, E.G. RNomics in Escherichia coli detects new sRNA species and indicates parallel transcriptional output in bacteria. Nucleic Acids Res. 2003, 31, 6435-6443.

13. Sittka, A.; Lucchini, S.; Papenfort, K.; Sharma, C.M.; Rolle, K.; Binnewies, T.T.; Hinton, J.C.; Vogel, J. Deep sequencing analysis of small noncoding RNA and mRNA targets of the global post-transcriptional regulator, Hfq. PLoS Genet. 2008, 4, e1000163.

14. Sittka, A.; Sharma, C.M.; Rolle, K.; Vogel, J. Deep sequencing of Salmonella RNA associated with heterologous Hfa proteins in vivo reveals small RNAs as a major target class and identifies RNA processing phenotypes. RNA Biol. 2009, 6, 266-275.

15. Sonnleitner, E.; Sorger-Domenigg, T.; Madej, M.J.; Findeiss, S.; Hackermuller, J.; Huttenhofer, A.; Stadler, P.F.; Blasi, U.; Moll, I. Detection of small RNAs in Pseudomonas aeruginosa by RNomics and structure-based bioinformatic tools. Microbiology 2008, 154, 3175-3187.

16. Kreikemeyer, B.; Klenk, M.; Podbielski, A. The intracellular status of Streptococcus pyogenes: role of extracellular matrix-binding proteins and their regulation. Int. J. Med. Microbiol. 2004, 294, 177-188.

17. Mangold, M.; Siller, M.; Roppenser, B.; Vlaminckx, B.J.; Penfound, T.A.; Klein, R.; Novak, R.; Novick, R.P.; Charpentier, E. Synthesis of group A streptococcal virulence factors is controlled by a regulatory RNA molecule. Mol. Microbiol. 2004, 53, 1515-1527.

18. Perez, N.; Trevino, J.; Liu, Z.; Ho, S.C.; Babitzke, P.; Sumby, P. A genome-wide analysis of small regulatory RNAs in the human pathogen group A Streptococcus. PLoS One 2009, 4, e7668.

19. Halfmann, A.; Kovacs, M.; Hakenbeck, R.; Bruckner, R. Identification of the genes directly controlled by the response regulator CiaR in Streptococcus pneumoniae: five out of 15 promoters drive expression of small non-coding RNAs. Mol. Microbiol. 2007, 66, 110-126.

20. Pichon, C.; Felden, B. Small RNA genes expressed from Staphylococcus aureus genomic and pathogenicity islands with specific expression among pathogenic strains. Proc. Natl. Acad. Sci. USA 2005, 102, 14249-14254.

21. Sorek, R.; Cossart, P. Prokaryotic transcriptomics: a new view on regulation, physiology and pathogenicity. Nat. Rev. Genet. 2010, 11, 9-16.

22. Morita, T.; Maki, K.; Aiba, H. RNase E-based ribonucleoprotein complexes: mechanical basis of mRNA destabilization mediated by bacterial noncoding RNAs. Genes Dev. 2005, 19, 2176-2186.

23. Gripenland, J.; Netterling, S.; Loh, E.; Tiensuu, T.; Toledo-Arana, A.; Johansson, J. RNAs: regulators of bacterial virulence. Nat. Rev. Microbiol. 2010, 8, 857-866.

24. Loh, E.; Dussurget, O.; Gripenland, J.; Vaitkevicius, K.; Tiensuu, T.; Mandin, P.; Repoila, F.; Buchrieser, C.; Cossart, P.; Johansson, J. A trans-acting riboswitch controls expression of the virulence regulator PrfA in Listeria monocytogenes. Cell 2009, 139, 770-779.

25. Chatterjee, S.S.; Hossain, H.; Otten, S.; Kuenne, C.; Kuchmina, K.; Machata, S.; Domann, E.; Chakraborty, T.; Hain, T. Intracellular gene expression profile of Listeria monocytogenes. Infect. Immun. 2006, 74, 1323-1338.

26. Joseph, B.; Przybilla, K.; Stuhler, C.; Schauer, K.; Slaghuis, J.; Fuchs, T.M.; Goebel, W. Identification of Listeria monocytogenes genes contributing to intracellular replication by expression profiling and mutant screening. J. Bacteriol. 2006, 188, 556-568. 
27. Hain, T.; Hossain, H.; Chatterjee, S.S.; Machata, S.; Volk, U.; Wagner, S.; Brors, B.; Haas, S.; Kuenne, C.T.; Billion, A.; et al. Temporal transcriptomic analysis of the Listeria monocytogenes EGD-e sigma B regulon. BMC Microbiol. 2008, 8, 20.

28. McGann, P.; Raengpradub, S.; Ivanek, R.; Wiedmann, M.; Boor, K.J. Differential regulation of Listeria monocytogenes internalin and internalin-like genes by sigma B and PrfA as revealed by subgenomic microarray analyses. Foodborne. Pathog. Dis. 2008, 5, 417-435.

29. Wang, Z.; Gerstein, M.; Snyder, M. RNA-Seq: A revolutionary tool for transcriptomics. Nat. Rev. Genet. 2009, 10, 57-63.

30. Wurtzel, O.; Sapra, R.; Chen, F.; Zhu, Y.; Simmons, B.A.; Sorek, R. A single-base resolution map of an archaeal transcriptome. Genome Res. 2010, 20, 133-141.

31. Barry, T.; Kelly, M.; Glynn, B.; Peden, J. Molecular cloning and phylogenetic analysis of the small cytoplasmic RNA from Listeria monocytogenes. FEMS Microbiol. Lett. 1999, 173, 47-53.

32. Nielsen, J.S.; Olsen, A.S.; Bonde, M.; Valentin-Hansen, P.; Kallipolitis, B.H. Identification of a sigma B-dependent small noncoding RNA in Listeria monocytogenes. J. Bacteriol. 2008, 190, 6264-6270.

33. Mandin, P.; Repoila, F.; Vergassola, M.; Geissmann, T.; Cossart, P. Identification of new noncoding RNAs in Listeria monocytogenes and prediction of mRNA targets. Nucleic Acids Res. 2007, 35, 962-974.

34. Christiansen, J.K.; Nielsen, J.S.; Ebersbach, T.; Valentin-Hansen, P.; Sogaard-Andersen, L.; Kallipolitis, B.H. Identification of small Hfq-binding RNAs in Listeria monocytogenes. RNA 2006, 12, 1383-1396.

35. Livny, J.; Teonadi, H.; Livny, M.; Waldor, M.K. High-throughput, kingdom-wide prediction and annotation of bacterial non-coding RNAs. PLoS One 2008, 3, e3197.

36. Johansson, J.; Mandin, P.; Renzoni, A.; Chiaruttini, C.; Springer, M.; Cossart, P. An RNA thermosensor controls expression of virulence genes in Listeria monocytogenes. Cell 2002, 110, 551-561.

37. Mansjo, M.; Johansson, J. The Riboflavin analog Roseoflavin targets an FMN-riboswitch and blocks Listeria monocytogenes growth, but also stimulates virulence gene-expression and infection. RNA Biol. 2011, 8, 674-680.

38. Christiansen, J.K.; Larsen, M.H.; Ingmer, H.; Sogaard-Andersen, L.; Kallipolitis, B.H. The RNA-binding protein $\mathrm{Hfq}$ of Listeria monocytogenes: role in stress tolerance and virulence. J. Bacteriol. 2004, 186, 3355-3362.

39. Vogel, J. A rough guide to the non-coding RNA world of Salmonella. Mol. Microbiol. 2009, 71, $1-11$.

40. Mraheil, M.A.; Billion, A.; Kuenne, C.; Pischimarov, J.; Kreikemeyer, B.; Engelmann, S.; Hartke, A.; Giard, J.C.; Rupnik, M.; Vorwerk, S.; et al. Comparative genome-wide analysis of small RNAs of major Gram-positive pathogens: from identification to application. Microb. Biotechnol. 2010, 3, 658-676.

(C) 2011 by the authors; licensee MDPI, Basel, Switzerland. This article is an open access article distributed under the terms and conditions of the Creative Commons Attribution license (http://creativecommons.org/licenses/by/3.0/). 